\title{
Crecimiento económico ligado al desarrollo del emprendimiento social
}

\section{Economic growth linked to the development of social entrepreneurship}

Méndez Bravo, J. C.

Universidad de Guayaquil, Ecuador

Quintanilla Arámbulo, E.A.

Universidad de Guayaquil, Ecuador

Sánchez Jaramillo, M. E.

Universidad de Guayaquil, Ecuador

Autor por Correspondencia: julio.mendezb@ug.edu.ec, elvis.quintanillaa@ug.edu.ec, maylin.sanchezj@ug.edu.ec

Fecha de recepción: 08 de Octubre 2017 - Fecha de aceptación: 15 de Febrero de 2018

\section{Resumen}

El presente trabajo exponemos el emprendimiento social como un modelo de negocio viable, considerando los aportes de diferentes autores en cuanto al logro del desarrollo de la misión y valor social al cual se comprometen estos emprendedores y el tipo de modelo en que se puede dividir un emprendimiento social conocido como emprendimiento social hibrido, un sector que se ve afectado positivamente con el desarrollo de este tipo de emprendimientos sociales es la economía, aquí se revisará brevemente cuales son los factores que impulsan o afectan al crecimiento económico, y como se relaciona el emprendimiento social desde la perspectiva económica.

Palabras Clave: emprendimiento social; crecimiento económico; cambio social; oportunidades; emprendimiento social hibrido

\begin{abstract}
In this paper we expose social entrepreneurship as a viable business model, considering the contributions of different authors in the achievement of the development of the mission and social value to which these entrepreneurs undertake and the type of model in which one can divide a social enterprise known as a hybrid social venture, a sector that is positively affected by the development of this type of social entrepreneurship is the economy, there will be a brief review of the factors that drive or affect economic growth, and how social entrepreneurship is related from the economic perspective.
\end{abstract}

Key words: social entrepreneurship; economic growth; social change; opportunities; hybrid social enterprise 


\section{Introducción}

El emprendimiento social se asocia a la actividad del sector sin fines de lucro, tal como lo menciona Light (2006) citado en (Silva , 2015)es una manera que favorece o promete soluciones a problemas como la pobreza, el hambre y las enfermedades. Los emprendimientos sociales desempeñan un papel importante desarrollando la iniciativa de cada individuo, en el ámbito económico Shane, Venkataraman (2000) y Dees (1998) entiende por emprendimiento la creación y consolidación de empresas a partir de la explotación de oportunidades resolviendo problemas sociales y económico (Santos, Barroso, \& Guzmán, 2013).

Para Porter y Kramer (2011), Mackey y Sisodia (2013) y Londres y Hart (2011), señalan que las empresas tradicionales no solo deben buscar beneficios sino buscar tener un propósito social que lograr una situación de ganar y ganar. (Barki, Comini, Cunliffe, Hart, \& Rai, 2015) Por otro lado, autores como Emerson \& Twersky (1996) definen al emprendimiento social como actividades innovadoras con un objetivo social en las empresas con o sin fines de lucro o estructuras híbridas con el fin que mencionan Reis (1999) y Thompson (2002) el cual es desarrollar enfoques innovadores para obtener ingresos para crear valor social en lugar de riqueza (Austin, Stevenson, \& Wei-Skilern, 2012).

Según Holcombe (2007), señala que "el crecimiento económico está relacionado con la cantidad de producto mientras que el progreso económico lo está con su calidad”. De ahí Schumpeter (1934) considera como factor importante el proceso innovador, el cual depende del empresario. (Galindo, Méndez, \& Castaño, 2016)

Durante el paso del tiempo se ha venido recolectando teoría de diferentes autores identificando factores comunes entre los cuales están el clima favorable hacia el emprendedor, las perspectivas económicas del país y las políticas económicas y de apoyo a la empresa, aparecen como fundamentales para el desarrollo de la actividad. (Lupiáñez , Priede, \& López, 2014)

Autores como Alvarez \& Barney (2007), Ardichvili (2003), declaran que existen dos perspectivas las cuales argumenta que las oportunidades son creadas por emprendedores a partir de los recursos que posee o son descubiertas por una necesidad. (Simón, Sastre, \& Revuelto, 2015). Para Becerra, Cortés, Malacara, \& Alegría (2014), el emprendimiento social son el resultado de iniciativas para cambiar la sociedad mediante el planteamiento de propuestas y creación de bienes o servicios que alcance un objetivo social con la cooperación de otros actores.

Declara Méndez, Galindo \& Ribeiro (2012), los países deben enfrentarse a los problemas económicos, desarrollando modelo de crecimiento económico que resuelvan problemas y persistan el futuro. (Galindo, Méndez, \& Castaño, 2016)

El emprendimiento social no solo busca la mejora de la calidad de vida mediante el crecimiento de la autoestima de los emprendedores ni el alivio de la conciencia social de los financistas, sino que busca un desarrollo y crecimiento de la calidad de vida mediante un desarrollo económico de los emprendedores y de una satisfacción de conciencia social de los financistas que va de la mano de un crecimiento de sus finanzas. 


\section{El emprendimiento social}

Las personas no pueden vivir sólo para satisfacer intereses personales sino también prestar atención a los intereses de los demás esta es la raíz del comportamiento del emprendedor social. (Sijabat, 2015). Shane (2000) señala 3 tipos de conocimientos previos relevantes para la identificación de oportunidades emprendedoras: el conocimiento de los mercados, las formas de servirlos y los problemas de los clientes, también se mencionan que esas oportunidades están relacionadas con su formación y experiencia laboral. (Simón, Sastre, \& Revuelto, 2015)

Según Martin \& Osberg (2007) citado en Sijabat (2015) sostienen que los empresarios sociales surgen debido a la pobreza, la marginación y la exclusión en la sociedad social. Los empresarios sociales hacen hincapié en el cambio social en lugar de ganancias. Que surgen como una reacción a las empresas que adoptan una tendencia orientada a la línea de fondo para buscar beneficios a corto plazo a costa de los beneficios a largo plazo para la sociedad. (Madhooshi \& Jafari, 2015).

Según Sekliuckiene \& Kisielius (2015) las empresas sociales y los empresarios sociales crean iniciativas innovadoras y soluciones a problemas sociales sin resolver, poniendo la creación de valor social en el centro de su misión con el fin de crear beneficios para diferentes individuos, comunidades y otros grupos. De una manera nueva que aumenta la conciencia pública sobre el problema a través de su visión, trabajo y actividades (Rahim \& Mohter, 2015).

Por ejemplo, Mort, Weerawardena \& Carnegie (2002) citado en Sijabat (2015), escribió que el emprendimiento social es multidimensional y engloba las actividades comerciales (empresarial) en el logro de la misión social. "El emprendimiento social es un concepto muy joven y es muy buscada fuera en el mundo práctico, sin embargo, todavía se considera en su fase inicial en la plataforma académica". (Johnson, 2002; Roberts y Woods, 2005) citado en (Rahim \& Mohter, 2015)

Para tener una ilustración teórica de que es el emprendimiento social según varios autores mencionan que el SE es "un proceso mediante el cual se puede crear valor social mediante la combinación de recursos de nuevas maneras, y estas combinaciones de recursos puede explorar y explotar las oportunidades que crean valor y estimular el cambio social." (Silva Niño, 2015). Tales cambios sociales son la contribución de los empresarios sociales en la mejora la calidad de vida, la eficiencia y la sostenibilidad del crecimiento económico y social.

(Popoviviu \& Popoviciu, 2011) citado en (Sijabat, 2015).

Menciona Sijabat (2015) que "El emprendimiento social es descrito como la acción de los individuos para el cambio utilizando principios y modelos empresariales para satisfacer las necesidades sociales, para hacer frente a los problemas sociales, y mantener los valores sociales". Otros autores afirman que los emprendedores sociales son individuos u organizaciones comprometidas en actividades de emprendimiento con un objetivo generar valor social. Bossman \& Livie (2010) citado en (Bargsted, 2013).

El emprendimiento social abarca las actividades y procesos realizados para descubrir, definir y explotar las oportunidades a fin de aumentar la riqueza social mediante la creación de 
nueva empresa o la gestión de las organizaciones existentes de una manera innovadora (Madhooshi \& Jafari Samimi, 2015)

"Se afirma que el emprendimiento social es la creación de riqueza, como un medio para un fin y las estructuras corporativas se utilizan para la creación de valor social " (Silvia, 2015). Según Santos (2012) citado en (Sijabat, 2015), menciona que el emprendimiento social es un proceso de innovación económica que se produce con una variedad de características de las instituciones sobre la base de la creación de valores en los que el enfoque utilizado es generalmente adecuada y apropiada para abordar los problemas de la sociedad moderna.

Para Maryam (2012), el emprendimiento social posee el propósito de crear beneficios para satisfacer fines sociales mediante estrategias con responsabilidad junta la participación de socios y partes interesadas para lograr condiciones de trabajos buenas y lograr el retorno de capital para incrementar los servicios con fines sociales y económicos. (Becerra, Cortés, Malacara, \& Alegría, 2014).

Shane \& Venkataraman (2000), fueron pioneros en proponer al emprendimiento como un proceso de identificación, evaluación y explotación de oportunidades tomando parte de los emprendedores en sus estudios. (Simón, Sastre, \& Revuelto, 2015)

Según Groot y Dankbaar (2014) citado en Sijabat (2015) La innovación social se refiere al descubrimiento de nuevas ideas relacionadas con productos, servicios y modelos que podrían utilizarse para fomentar el cumplimiento de las necesidades sociales tales como la inclusión social, la protección del medio ambiente y el desarrollo sostenible, así como el establecimiento relación y nueva colaboración social.

Thornton, Ribeiro \& Urbano (2011) considera al gobierno, instituciones, cultura y políticas como un papel importante en el desempeño económico ya que estas determinan la conducta de agentes económicos que aseguran el progreso. (Galindo, Méndez, \& Castaño, 2016)

Como sabemos el emprendimiento es innovación el cual nos permite abrir caminos de crecimiento para lo cual la Universidad, Gobierno, Comunidad y Empresas del entorno son necesarias para generar múltiples alternativas productivas (Salinas \& Osorio, 2012). Por lo tanto, "la innovación social está estrechamente relacionado con el emprendimiento social, donde el empresario social es el actor de la innovación social" ( Sijabat, 2015). De acuerdo con Grisolia y Ferrangina (2015) citado en Kazmi, Hashim, \& Hung (2016) Innovaciones sociales se definen como "nuevas ideas (productos, servicios y modelos) que simultánea se corresponde con necesidades sociales y crear nuevas relaciones sociales."

Al involucrar el sector social en estos procesos de emprendimiento, se fortalecen los niveles de competitividad empresarial, contribuyendo al desarrollo de la región o del país, así queda demostrado, que las empresas creadas desde el enfoque social, genera no sólo una forma de empleo y de concepción de la empresa, también brinda bienestar a la comunidad involucrada reduciendo los niveles de exclusión social (Salinas \& Osorio, 2012). "Para que el emprendimiento social se desarrolle y alcance mayor resultados para la zona, es necesario 
integrar varios factores de manera apropiada, como plantear estrategias para acceder apoyos y programas gubernamentales e institucionales" Silva (2007) citado en (Becerra, Cortés, Malacara, \& Alegría, 2014)

No obstante, un emprendimiento debe contar con las facilidades que permiten su creación dentro del marco legal y estas sean apropiadas para no obstaculizar la visión de un joven emprendedor social, por esa razón Salinas \& Osorio (2012), propone simplificar los trámites, reducir las barreras y los costos, suavizar la legislación y promover el espíritu empresarial en la escuela, apoyarse en la universidad y acceso a préstamos.

El fortalecimiento del emprendimiento es determinante como elemento transversal en la formación de personas aptas para incorporarse en las dinámicas del conocimiento y desarrollo socioeconómico de países con profundas necesidades de cambio. Castellanos (2003) citado en (Bouby , 2015). Permitiendo actuar en áreas donde ante no solía hacerse creando un sistema más justo y logrando oportunidades de desarrollo. Kliksberg (2011), citado en (Radrigan, Dávila, \& Penaglia, 2012) se asegura que "el tema formativo es decisivo para el futuro de los emprendimientos sociales, asimismo afirma que, proporcionar en forma sistemática, a través de instituciones especializadas, y del mundo de las universidades, una formación sólida, es uno de los desafíos centrales para su avance.".

Mejorar la formación del capital humano según Sylwester (2000), es indispensable por el simple hecho que desempeñan un papel importante en el proceso, además su calidad, eficiencia y necesidad de entorno social buscan que estos estén mejor preparado para el mercado meta. (Galindo, Méndez, \& Castaño, 2016)

Según García y Piña(2013) citado en (Bouby , 2015) destaca que se debe crear un entorno para fomentar el emprendimiento social, constituido principalmente por normas jurídicas y políticas públicas orientadas a priorizar la integración de los aspectos económicos y sociales con alta participación de organizaciones comunitarias y fuerte intervención estatal en las áreas financiera, técnica y de capacitación, con el propósito de alcanzar el desarrollo endógeno y humanista como modelo de diversificación de la economía, bajo parámetros de sustentabilidad.

"Los emprendimientos sociales se desarrollan en cuatro etapas, comenzando como iniciativa de un individuo quien, al identificar un problema de orden social, económico o ambiental, desarrolla un proyecto para contribuir a su solución" (Radrigan, Dávila, \& Penaglia, 2012). No todo puede llamarse emprendimiento social, esto demuestra que este tipo emprendimiento tiene como finalidad a contribuir a la resolución de un problema que este dentro de lo social, económico o ambiental.

Tal como lo menciona Jurgita Sekliuckiene (2015) en su análisis sobre el emprendimiento social, los emprendedores son influenciado por el entorno y lo estimulan a tomar iniciativas, ya que este se está transformando en un nuevo mercado.

Diferentes autores para identificar los factores detonantes del emprendimiento social analizaron y desarrollaron investigaciones que lograron identificar las claves para el éxito entre 
ellas estaban la financiación que poseen, su campo de actividad, su perfil personal, objetivos y estrategias de crecimiento, a estos se agregó las políticas que rigen sobre el país, la situación económica que atraviesa en la actualidad. (Becerra, Cortés, Malacara, \& Alegría, 2014)

\section{Un nuevo modelo de emprendimiento social}

"Los empresarios sociales son los precursores del cambio, ideando nuevas formas de proporcionar apoyo y desarrollo para los excluidos de las oportunidades de la nueva sociedad" (Handy, 1997; Lob \& Mohter, 2015)." Las empresas sociales son necesarias para el desarrollo de nuevos modelos para los tiempos presentes y futuros, ya que combinan la pasión de una misión social con la disciplina, la innovación y la gestión de una empresa" (Silva, 2015).

Las empresas sociales pueden ser clasificados por su orientación misión, por el nivel de integración entre los programas sociales sin ánimo de lucro y con fines de lucro comercial, y por sus mercados objetivos previstos (Alter,kasmi 2006; Grassl, 2012).

La idea de organización híbrida que se conoce como la empresa social o de riesgo social, así (Dorado, 2006; Townsend \& Hart, 2008; Lob \& Mohter, 2015). Este tipo de emprendimiento social se centra en la mezcla de objetivos comerciales y sociales (Dees, 1998; Dorado, 2006; Townsend \& Hart, 2008; Lob \& Mohter, 2015).

En los modelos híbridos de negocios, el espíritu empresarial es la agencia de síntesis que hace que los elementos dispares se unen. (Grassl, 2012). Para comprender mejor el emprendimiento social, se ha creado un modelo de emprendimiento social en el cual se detalla el emprendimiento social hibrido. "El emprendimiento social se clasifica en dos categorías diferentes; sin ánimo de lucro e híbridos (una organización con objetivos financieros y sociales) organizaciones" (Rahim \& Mohter, 2015).

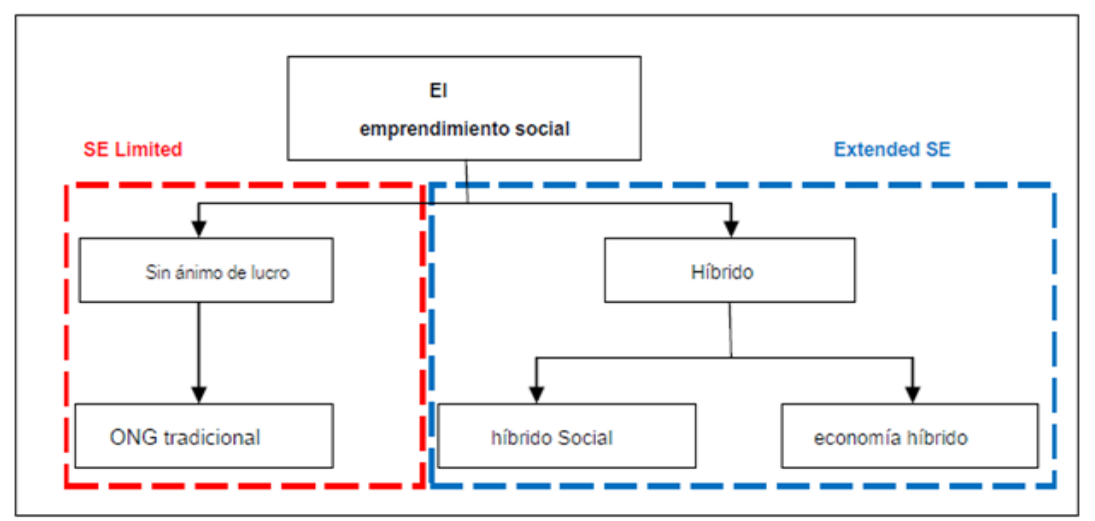

Figura 1: Modelo de Emprendimiento Social. Fuente: Rahim \& Mohter (2015)

Se puede destacar en la figura el tipo de modelo de emprendimiento social por el que se puede optar al momento de crear un emprendimiento, desde un punto de vista organizaciones que captan ingresos para cubrir las necesidades insatisfechas del ámbito social y por otro lado organizaciones que buscan maximizar los ingresos para la obtención de ganancia y satisfacer las necesidades sociales de su objetivo de emprendimiento social.

Terjesen et al. (2011) citado en Rahim \& Mother (2015) ha realizado un estudio denominado "Informe Global Entrepreneurship Monitor de Emprendimiento Social”. En su 
estudio, se hicieron entrevistas con 150.000 adultos en 49 países durante el año 2009, y se documentan la prevalencia de emprendimiento social en una población por medio de una encuesta estandarizada en cada uno de los países.

Tabla 1: Emprendimiento Social Tasas de prevalencia.

\begin{tabular}{|l|c|c|}
\hline Rogión & $\begin{array}{c}\text { Sin fines de lucro SE } \\
(\%)\end{array}$ & $\begin{array}{c}\text { SE Economicamente } \\
\text { hibrido (\%) }\end{array}$ \\
\hline Europa Oriental & 1.1 & 1.0 \\
\hline Europa del Este & 0.9 & 1.1 \\
\hline America latina & 0.8 & 1.6 \\
\hline El sudeste de Asla & 0.4 & 1.0 \\
\hline Oriente Medio y el Norte de Africa & 0.8 & 0.8 \\
\hline caribe & 1.4 & 2.8 \\
\hline Africa & 0.7 & 0.7 \\
\hline Estados Unidos & 2.3 & 1.4 \\
\hline Promedio & 1.05 & 1.30 \\
\hline
\end{tabular}

Fuente: Rahim \& Mohter (2015)

Dentro de todo el estudio se ha determinado que las organizaciones de emprendimiento social económicamente híbridos poseen una tasa de prevalencia más alta (1.30) a diferencia de las organizaciones social sin fines de lucro (1.05). Esto da a conocer que en todas las regiones del mundo existe una alta aceptación de organizaciones de emprendimiento social económicamente híbridos, excepto en Europa Occidental y los Estados Unidos de América que optan por los emprendimientos sociales sin fines de lucro. Lo que establece que el desarrollo de emprendimientos sociales con fines de lucro no solo para los emprendedores sino para los mentalizadores y financistas tendría un mayor efecto positivo en las sociedades donde se desarrollen dichos emprendimientos.

\section{Factores que influyen en el crecimiento económico}

El crecimiento económico es una medida del bienestar de un país y su población. Según Kutznets (1966) citado en (Galindo M. Á., 2011) "es un incremento sostenido del producto por capital o por trabajador", lo cual sería el aumento del valor de los bienes y servicios producido por una economía durante un periodo determinado.

El impulsar el crecimiento económico permitirá disponer de mayor números de bienes y servicios, empleos en fin mejorar el bienestar y el clima social para el futuro por tal motivo el emprendedor juega un papel importante no solamente descubriendo nuevas oportunidades aprovechando los recursos existente sino también en el impulsar de emprendimiento de tercero generando efecto indirectos que puede afectar o beneficiar de la forma en que lo plantee tal como producto similares o productos complementarios al nuestro.(Galindo M. Á., 2011)

"El crecimiento económico se puede ver reflejado mediante las actitudes, desafíos y oportunidades que generan los jóvenes emprendedores, pero puede verse perjudicada por entrar en una fase de ralentización poniendo en riesgos los avances sociales políticos y económicos" (OCDE, CEPAL, \& CAF, 2016). 
Esfehani \& Ramírez citado en López \& Apolinario (2015) menciona que la Inversión Pública lo que hace es crear las condiciones mínimas necesarias para que la demanda de bienes y servicios producidos por el sector privado se incrementen y de esa forma los inversionistas privados puedan hacer formación racional de expectativas futuras sobre sus ventas y rendimientos futuros.

El asumir riesgos, defendida por Knight (1921) y apoyada por Galindo (2011) consideran que asumir la incertidumbre puede llegar a confirmar riesgo existente que produzcan cambios negativos. Tal como lo menciona Tsiddon (1992), se debe evitar las restricciones en el mercado que dificulten el acceso de obtención de recursos. (Galindo, Méndez, \& Castaño, 2016) Schumpeter (1934) considera como factor relevante el proceso innovador, que desde su perspectiva, dependerá del empresario y del avance tecnológico en los procesos productivos los cual lleva a la existencia de emprendedores. (Galindo, Méndez, \& Castaño, 2016)

Fabio Manca citado en Aragón, Arroyo, \& Figueroa (2014), muestra evidencia de complementariedades entre la inversión del sector público en I+D y la acumulación de capital humano, las cuales tienen un impacto significativo en el crecimiento de las regiones. Sus resultados ponen de manifiesto la importancia del capital humano en el proceso de crecimiento económico en las regiones europeas así como también que la I+D desempeña un papel positivo en el crecimiento económico de las mismas.

Los factores que impiden el crecimiento económico que se han vistos reflejados en diferentes países como Argentina, Brasil, Chile, Colombia y Costa Rica se identificaron las condiciones del mercado, la falta de empleo, el abandono de estudios superiores entre los jóvenes, la alta tasa de desempleo, aquellos que poseen empleo no reciben capacitaciones, falta de involucración por parte del Estado, problemas con la complejidad de los procedimientos regulatorios para nuevas empresas. (OCDE, CEPAL, \& CAF, 2016)

Los factores de éxito entre los emprendimientos sociales que se identificaron en Chile y América Latina se encontraron el apoyo de agentes externos, alianzas estratégicas, múltiples fuentes de financiamiento, una adecuada planificación y flexibilidad a los cambio, conocimientos y aptitudes de administración, equipo comprometido, diagnóstico del problema social, innovación y participación dela comunidad. (Becerra, Cortés, Malacara, \& Alegría, 2014)

El crecimiento económico sostenible es una preocupación predominante para todas las economías, especialmente para las economías en desarrollo, en los niveles iniciales, las entradas de deuda externa ofrecen cierta facilidad en el frente fiscal y ayudan a las economías a crecer. Sin embargo, los mayores desequilibrios fiscales, el uso inadecuado de estos recursos y la reprogramación de la deuda externa conducen a un mayor nivel de deuda externa ya un servicio creciente de la deuda. La fuerte deuda externa no implica necesariamente un lento crecimiento económico, la incapacidad de un país para cumplir con sus obligaciones de deuda la agrava. (Shabbir, 2013)

Para López (2015) un factor determinante del crecimiento es la inversión pública por su capacidad de producir bienes que el sector privado no podría proveer sin asumir elevados costos 
de operación y con ello limitar la posibilidad de adquisicion de otros agentes, dado que se debe colocar precios altos para generar beneficios que satisfagan la alta inversión inicial.

\section{El emprendimiento social desde la perspectiva económica}

"El emprendimiento social es vital para desbloquear la inclusión económica y el crecimiento de las economías en desarrollo (Kazmi, Hashim, \& Hung, 2016). Según Salinas \& Osorio (2012) El emprendimiento visto desde la economía, es cuando la persona identifica oportunidades con la finalidad de alcanzar beneficio e impacto en la sociedad, para lo cual debe arriesgar dentro de la eficacia y eficiencia. Según Bouby (2015), el emprendimiento social está surgiendo como una respuesta a los problemas que está generando el modelo de capitalismo global a la población, promoviendo la competitividad de las empresas mediante del reforzamiento y apoyo de políticas.

El emprendimiento social es un modelo empresarial complementario al emprendimiento económico o de mercado, al actuar en áreas donde no suele actuar el emprendimiento de mercado, por lo cual puede contribuir a mejorar el sistema económico global en un sistema más justo y eficiente. Yunus citado en (Bouby, 2015).

Las competencias y habilidades le han permitido desarrollar una mayor capacidad de adaptación y mejor inserción a los emprendedores social en el mercado, entre estas sobresalen sus diversos conocimientos adquiridos, experiencias, creatividad, trabajo en equipo, compromiso, liderazgo y comunicación favorecen a establecer un emprendimiento con base solidas capaz de aportar al crecimiento económico. "cada emprendedor social debe movilizar recursos para lograr sus objetivos" (Madhooshi \& Jafari, 2015)

Empresarios, entre ellos empresarios sociales, actúan como agentes de cambios importantes en la economía, y por lo tanto se convierten en agentes de crecimiento económico y de empleo y la creación de riqueza que pueden mejorar el bienestar de la comunidad. Wiklund, Davidsson, Audretsch \& Karlsso (2011) citado en (Sijabat, 2015).

Hilde Schwab (2011) citado en Kazmi, Hashim, Hung \& Ullah (2016) dijo: "Los empresarios sociales y sus innovaciones promover el crecimiento económico inclusivo." según Urama (2013) citado en Kazmi, Hashim, Hung \& Ullah (2016)" En todo el mundo durante la última década, ha habido un aumento fenomenal de interés en las innovaciones sociales como una forma de lograr un crecimiento económico sostenible".

Las actividades económicas son uno de los elementos clave para el emprendimiento social "Actividades de Emprendimiento Social tienen un impacto económico en las comunidades (que están involucrados) y la organización empresarial en estado puro" (Duff,

2011; Kazmi,Hashin,Hung \& Ullah, 2016 ).

\section{Conclusión}

El desarrollo de emprendimientos sociales híbridos no solo genera un beneficio económico y en la calidad de vida de los emprendedores y de los financistas, sino también tienen 
un efecto de bienestar en las sociedades y comunidades donde se desarrollan dichos emprendimientos, las medidas que deberían de tomarse para lograr establecer dicha situación es el involucramiento del estado mediante el incentivo de emprendedores y de la empresa privada en generar este tipo de emprendimientos. Lográndolo mediante un aumento en el nivel de educación mediante promoción de becas que motiven al terminar estudios superiores o desarrollar nuevas carreras que amplié el campo de acción de los emprendedores, ofrecimiento de capacitación a los empleadores y a trabajadores, como también a los potenciales emprendedores siendo estas impartidas por empresarios experimentados, el estado debe promover leyes enfocado a los emprendedores y jóvenes que buscan lanzar sus proyectos empresariales que cuenten con subsidios e incentivos o la reducción de trámites regulatorios para las nuevas empresas, promoviendo emprendimientos sociales destinados al mejoramiento de la calidad de vida de la sociedad.

\section{Bibliografía}

Aragón , A., Arroyo, T., \& Figueroa, J. (2014). El capital humano y la inversión en Investigación y Desarrollo (I+D) en el crecimiento ecónomico de la región noroeste de México. Revista Iberoamericana de Ciencias, (p. 96 - 108).

Austin, J., Stevenson, H., \& Wei-Skilern, J. (2012). Social and commercial entrepreneurship: same, different, or both? Revista de Administração - RAUSP, (p. 370 - 384).

Barki, E., Comini, G., Cunliffe, A., Hart, S., \& Rai, S. (2015). Social Entrepreneurship And Social Business: Retrospective And Prospective Research. RAE - Revista de Administração de, (p. 380 - 384).

Becerra, M. E., Cortés, E. M., Malacara, A. R., \& Alegría, N. L. (2014). Factores Detonadores del Emprendimiento Social, Base para el Análisis de Casos de Éxito. International Review of Business Research Papers, (p. 134 - 156).

Bouby , F. (2015). La Importancia Del Emprendimiento Social Frente Al Proceso De Globalizacion De La Economia Y Los Mercados. Rev. de Investigación de la Fac. de Ciencias Administrativas, UNMSM, (p. 39 - 49).

Galindo, M. Á. (2011). Crecimiento Económico. Revista Economía ICE, (p. 39 - 55).

Galindo, M., Méndez, M., \& Castaño, M. (2016). Crecimiento, progreso económico y emprendimiento. Journal of Innovation \& Knowledge, (p. 62 - 68).

Grassl, W. (2012). Modelos de negocio de la empresa social: Un enfoque de diseño de hibridación. ACRN Diario del espíritu empresarial Perspectives, (p. 37 - 69).

Kazmi, S., Hashim, M., \& Hung, D. M. (2016). Social Entrepreneurship and its Impact on Economy: In Perspective of Pakistan. International Journal of Academic Research in Accounting, Finance and Management Sciences, (p. 161 - 166). 
López, J. (2015). El Impacto De La Inversión Pública Sobre El Crecimiento Económico. Una Revisión Del Caso Ecuatoriano. Revista eumed.net, (p. 1 - 14).

Lupiáñez , L., Priede, T., \& López, C. (2014). El Emprendimiento Como Motor Del Crecimiento Económico. Revista ICE, (p. 55 - 64).

Mehrdad , M., \& Jafari Samimi, M. H. (2015). Social Entrepreneurship \& Social Capital: A Theoretical Analysis. American Journal of Economics, Finance and Management, (p. 102 - 112).

OCDE, CEPAL, \& CAF. (2016). Perspectivas económicas de América Latina 2017: Juventud, competencias y emprendimiento. OECD Publishing, (p. 1 - 338).

Radrigan, M., Dávila, A., \& Penaglia, F. (2012). Gestión y Liderazgos en los Emprendimientos Sociales: El caso del Sector No Lucrativo Chileno. Polis. Revista de la Universidad Bolivariana, (p. 141 - 165).

Rahim, L., \& Mohter, S. (2015). Social Entrepreneurship: A Different Perspective. International Academic Research Journal of Business and Technology, (p. 9 -15).

Salinas Ramos, F., \& Osorio Bayter, L. (2012). Emprendimiento y Economía Social, oportunidades y efectos en una sociedad en transformación. CIRIEC-España, revista de economía pública, social y cooperativa, (p. 130 - 151).

Santos, F., Barroso, M., \& Guzmán, C. (2013). La economía global y los emprendimientos sociales. Revista de Economía Mundial, (p. 177 - 196).

Sekliuckiene, J., \& Kisielius, E. (2015). Development of social entrepreneurship initiatives: a theoretical framework. ELSEVIER, 1015 - 1019.

Shabbir, S. (2013). Does External Debt Affect Economic Growth: Evidence from Developing Countries. SBP Working Paper Series, 1- 20.

Sijabat, R. (2015). The Role of Social Entrepreneurship in Enabling Economic Opportunities for the Poor: A Synthesis of the Literature and Empirical Works. International Journal of Business and Social Science, (p. 35 - 42).

Silva, A. C. (2015). Social Entrepreneurship and Corporate Social Responsibility: Differences and Points in Common. Journal of Business \& Economic Policy, (p. 86 - 98).

Simón, V., Sastre, O., \& Revuelto, L. (2015). El emprendedor social: análisis de la alerta social. ELSEIVER, (p. 155 - 165). 